\title{
Nanoscale
}

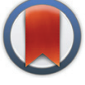

CrossMark \& click for updates

Cite this: Nanoscale, 2015, 7, 15080

Received 24th June 2015, Accepted 27th August 2015

DOI: $10.1039 / c 5 n r 04208 a$

www.rsc.org/nanoscale

\section{Location-specific nanoplasmonic sensing of biomolecular binding to lipid membranes with negative curvature $\uparrow$}

\author{
Juliane Junesch, ${ }^{a}$ Gustav Emilsson, ${ }^{a}$ Kunli Xiong, $\star^{a}$ Shailabh Kumar, ${ }^{b}$ \\ Takumi Sannomiya, ${ }^{c}$ Hudson Pace, ${ }^{a}$ Janos Vörös, ${ }^{d}$ Sang-Hyun Oh, ${ }^{b}$ Marta Bally ${ }^{a}$ and \\ Andreas B. Dahlin*a
}

\begin{abstract}
The biochemical processes of cell membranes are sensitive to the geometry of the lipid bilayer. We show how plasmonic "nanowells" provide label-free real-time analysis of molecules on membranes with detection of preferential binding at negative curvature. It is demonstrated that norovirus accumulate in invaginations due to multivalent interactions with glycosphingolipids.
\end{abstract}

Biosensors based on plasmonic nanostructures have been a subject of intense research for over a decade. In most cases detection is refractometric, i.e. the local increase in refractive index due to molecular binding to the surface induces a spectral redshift in the resonance, which enables label-free realtime analysis of biomolecular interactions by optical spectroscopy. ${ }^{1}$ Nanoplasmonic sensors based on single nanoparticles can even reach single molecule resolution in certain cases, ${ }^{2}$ which could prove useful when detecting targets from extremely limited sample quantities. ${ }^{3}$ However, due to its high resolution in terms of surface coverage and the possibility to detect small molecules, the established surface plasmon resonance (SPR) biosensor technology, ${ }^{4}$ which operates by refractometric detection on a planar metal surface, remains the golden standard for probing biomolecular interactions judging from the many SPR instruments now commercially available and the high number of studies generated every year. ${ }^{5} \mathrm{~A}$ hitherto unexplored path for making nanoplasmonic sensors

\footnotetext{
${ }^{a}$ Department of Applied Physics, Chalmers University of Technology, 41296 Göteborg, Sweden.E-mail: adahlin@chalmers.se

${ }^{b}$ Department of Electrical and Computer Engineering, University of Minnesota, 55455 Minneapolis, USA

${ }^{c}$ Department of Innovative and Engineered Materials, Tokyo Institute of Technology, 226-8503 Yokohama, Japan

${ }^{d}$ Department of Information Technology and Electrical Engineering, Swiss Federal Institute of Technology, 8092 Zürich, Switzerland

$\dagger$ Electronic supplementary information (ESI) available: Additional plasmonic sensing results, numerical electromagnetic simulations, quartz crystal microbalance data, fluorescence recovery after photobleaching, additional electron microscopy images, experimental methodology and materials used. See DOI: $10.1039 / \mathrm{c} 5 \mathrm{nr} 04208 \mathrm{a}$

$\$$ These authors contributed equally and substantially.
}

more useful in molecular biology (and elsewhere) is to use the nanostructures themselves to answer questions related to nanoscale geometry and how it influences binding affinity. Information on binding location is in principle accessible since different plasmon modes have a sensitivity that varies with position in the nanostructure. ${ }^{6}$ One type of questions that could be addressed is the influence from surface curvature, for instance when measuring interactions on lipid membranes, where the geometry plays an essential role for many fundamental biochemical processes. ${ }^{7-13}$ When investigating the influence from positive membrane curvature, lipid vesicles of different sizes can sometimes be used ${ }^{8}$ or possibly membrane coated plasmonic nanoparticles. ${ }^{14}$ However, negative membrane curvature implies major problems as the interior of a lipid vesicle is normally inaccessible. One option is to use lipid nanotubes created by manual micromanipulation of giant vesicles,${ }^{10}$ but one must then find means to load molecules of interest into the nanotube interior. In addition, detection requires fluorescent labeling. ${ }^{13}$ To overcome these limitations we have developed a sensor based on plasmonic "nanowells"15 coated with a continuous lipid membrane. The sensor uses the nanowells to mimic nanoscale invaginations or tubules found at the membrane cell and through a location-specific analysis of the spectral response, our sensor has the capability to directly detect whether binding preferentially occurs inside such structures. This is in addition to label-free and real-time operation with standard transmission spectroscopy using visible to near-infrared light. As an application example, we use the sensor to study how capsids ${ }^{16}$ (virus-like particles, VLPs) of non-enveloped gastroenteritis-causing norovirus ${ }^{17}$ binding to glycosphingolipids ${ }^{18}$ respond to membrane invaginations with a negative radius of curvature comparable to the size of the VLPs. The influence from multivalent interactions and surface diffusivity is further analyzed with a model system based on colloidal particles.

Nanowells, i.e. holes in two thin films consisting of gold and niobia $\left(\mathrm{Nb}_{2} \mathrm{O}_{5}\right)$ on a glass support, were fabricated by mask-on-metal colloidal lithography as described previously. ${ }^{15,19}$ 
The wells were selected to be $\sim 100 \mathrm{~nm}$ in diameter, the gold film $30 \mathrm{~nm}$ and the niobia $200 \mathrm{~nm}$ with $\sim 10$ wells per $\mu \mathrm{m}^{2}$ in short-range order over large areas. Note that the nanowell walls are not vertical (more images in $\mathrm{ESI}_{\dagger} \dagger$ ) so that the degree of negative surface curvature increases towards the bottom. For lipid membrane experiments (see below), we also deposited $15 \mathrm{~nm}$ silica $\left(\mathrm{SiO}_{2}\right)$ by atomic layer deposition, which gives a homogenous and conformal coating (Fig. 1a) that follows the surface contour. ${ }^{20}$

The plasmonic nanowells (and similar structures containing short-range ordered nanoscale apertures in a thin gold film) exhibit an asymmetric resonance showing a peak and a dip in the extinction spectrum ${ }^{21}$ (Fig. 1b). Previous work has shown that the spectral peak corresponds to grating-type coupling to propagating surface plasmons in the gold film, ${ }^{22}$ while the dip resonance has a field enhancement more focused to the interior of the void. ${ }^{15}$ The "bump" slightly below $600 \mathrm{~nm}$ is due to Fabry-Perot interference in the niobia ${ }^{15}$ and the bump slightly below $1000 \mathrm{~nm}$ is attributed to a water vibration mode. The general increase in extinction at shorter $(\sim 500 \mathrm{~nm})$ and longer $(\sim 1000 \mathrm{~nm})$ wavelengths is due to interband transitions in gold and reflection from the film respectively.

We hypothesized that the nanowells can be used as an optical nanoscale location-specific sensor by simultaneously monitoring the resonance wavelengths for both the peak $\left(\lambda_{\text {peak }}\right)$ and the dip $\left(\lambda_{\text {dip }}\right)$ by two centroid trackings. ${ }^{23}$ Although $\lambda_{\text {dip }}$ has a higher refractometric sensitivity than $\lambda_{\text {peak }}$ on average, ${ }^{15}$ the ratio of $\Delta \lambda_{\text {dip }}$ over $\Delta \lambda_{\text {peak }}$ is expected to be amplified inside the nanowells due to the nature of the resonances (propagating $v s$. localized). We first investigated this effect by material-selective chemistry ${ }^{24}$ on nanowells not coated with silica (Fig. 2). The surface chemistry was further verified independently using quartz crystal microbalance (ESI $\dagger$ ). As an initial test, we let the protein avidin adsorb everywhere ( $\mathrm{Au}$, $\mathrm{Nb}_{2} \mathrm{O}_{5}$ and glass), which gave approximately twice as high signal in $\lambda_{\text {dip }}$ compared to $\lambda_{\text {peak }}$ (Fig. 2a). This is expected since the average evanescent field extension is about the same for $\lambda_{\text {dip }}$ and $\lambda_{\text {peak }}{ }^{25}$ and thus the ratio of the signals in the parameters reflects that of the liquid "bulk sensitivities"15 (about twice as high for $\left.\lambda_{\text {dip }}\right)$. As another test for location-specific detection, selective binding of thiolated oligo(ethylene glycol) (OEG) to gold was monitored, which gave a signal in $\lambda_{\text {peak }}$ almost equal to that of $\lambda_{\text {dip }}$ (Fig. 2b). This is in agreement with the propagating surface plasmon mode having a sensitivity to refractive index changes primarily on the planar gold surface. Finally, selective protein adsorption inside nanowells enabled by the inert OEG coating gave a clear signal in $\lambda_{\text {dip }}$ while the change in $\lambda_{\text {peak }}$ was extremely low (Fig. 2c), showing that the surface plasmon is not sensitive to the refractive index in the interior of the nanowells. This remarkably high contrast in the peak signal depending on binding location should be highly suitable for detecting if binding occurs inside nanowells, as outlined in Fig. 2d. Based on the results we could define the parameter $\zeta=1-\Delta \lambda_{\text {peak }} / \Delta \lambda_{\text {dip }}$ as a measure of the degree of molecular localization to the interior of the nanowells: $\zeta$ is approximately zero for binding to the planar surface, 0.5 for binding without location preference and approaches one for binding down inside nanowells. Numerical simulations of the extinction spectrum of nanowells and its changes due to molecular binding obtained from standard electromagnetic theory and literature values of permittivity ${ }^{26}$ were consistent with the experimental results (ESI $\dagger$ ).

Inspired by the possibility to obtain information about where in the nanostructure binding occurs, we investigated the influence from membrane geometry for norovirus binding, which occurs via the esablishement of multivalent interactions to glycosphingolipids in the membrane. A continuous lipid bilayer with nanoscale invaginations was formed by exposing silica coated nanowells to unilamellar extruded lipid vesicles in a buffer solution. Bilayer formation was verified by following the characteristic kinetics of the plasmonic signal due to vesicle rupture ${ }^{27,28}$ and by fluorescence recovery after photobleaching $^{20}$ (ESI $\left.\dagger\right)$. Note that the membrane cannot span across the nanowells under these conditions, but follows the
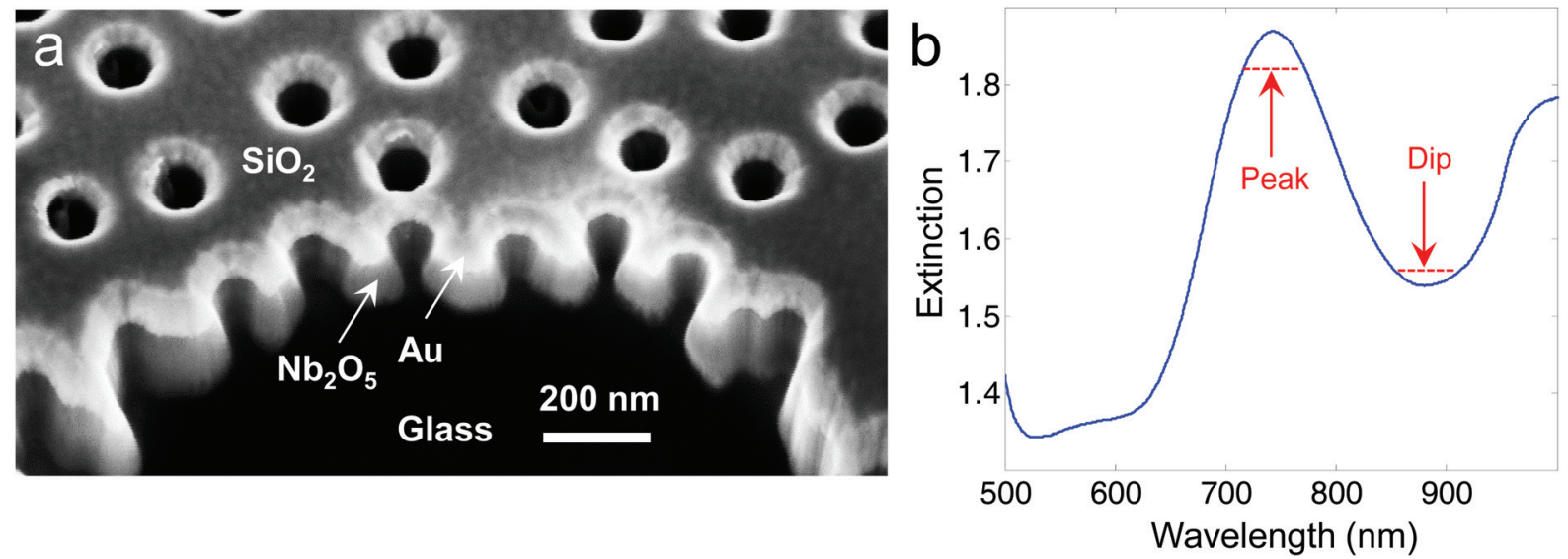

Fig. 1 Silica coated plasmonic nanowells. Scanning electron microscopy image of silica coated nanowells imaged at a scratched part of the sample under tilt (a). The extinction spectrum of silica coated nanowells in water, showing the plasmon resonance peak and dip (b). 

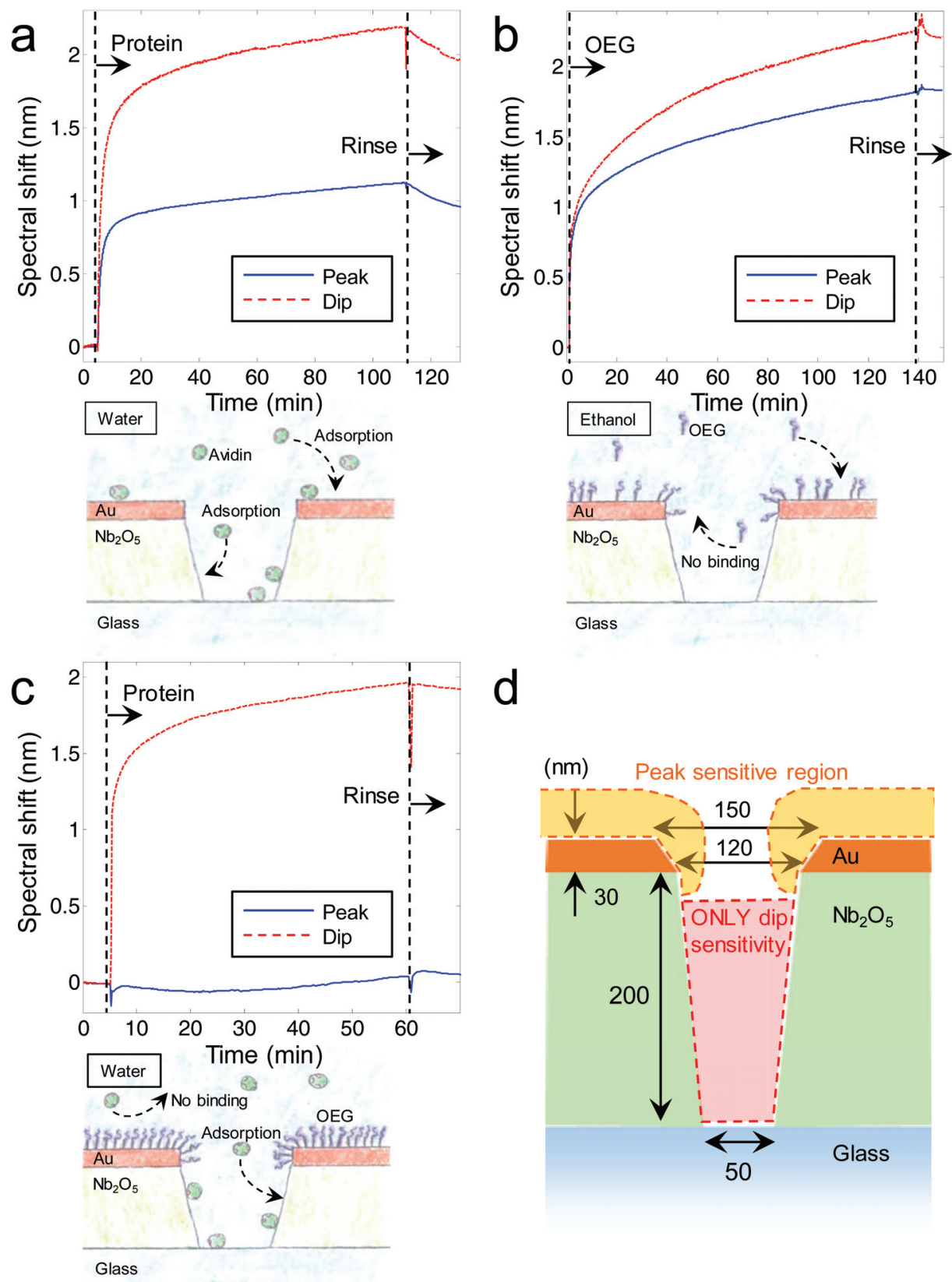

(nm) Peak sensitive region

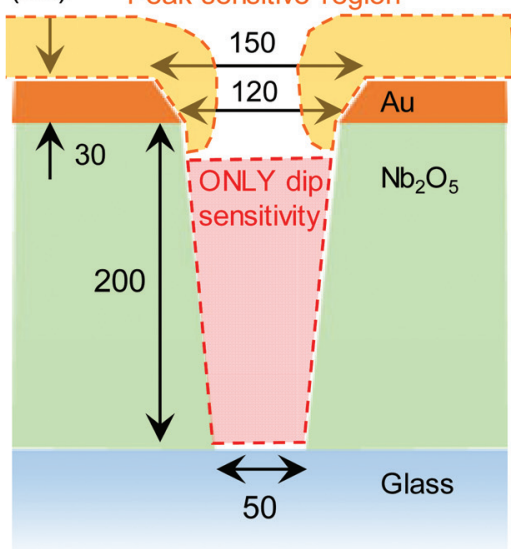

Fig. 2 Location-specific plasmonic detection with nanowells without silica coating. The signals in resonance wavelengths for peak and dip are monitored for direct protein adsorption (a), thiolated oligo(ethylene glycol) chemisorption to gold (b) and selective protein adsorption inside nanowells (c). (Note that for these experiments it is the ratio between the signals which should be compared and not their absolute values.) The nanowell shape and the different sensitivity distributions for peak and dip are also schematically illustrated (d).

surface contour. ${ }^{7,27}$ This is guaranteed by the fact that the vesicles are smaller than the apertures. ${ }^{29}$ (Membranes spanning across apertures are generally much more difficult to form.) Kinetics of binding of $\sim 40 \mathrm{~nm}$ VLPs (Spanish norovirus isolate Ast6139/01/Sp, ${ }^{17}$ recombinantly expressed ${ }^{30,31}$ ) to glycosphingolipids (histo-blood group antigen $\mathrm{B}$ type 1 ceramide $^{18,32}$ ) were monitored for different concentrations (Fig. 3a). VLP binding was found to be irreversible (no release upon rinsing) and no binding was observed to nanowells covered with mem- branes without glycosphingolipids as expected. ${ }^{33}$ Due to the multivalent nature of the interaction between a VLP and glycosphingolipids, ${ }^{18}$ it should accumulate inside the nanowells via surface diffusion since this will enable contact with more receptors, given that the VLP is comparable in size to the radius of negative curvature of the membrane. At the glycosphingolipid concentration used (5\%), the VLPs are expected to have relatively low mobility due to "standstill periods" on the order of minutes. ${ }^{16}$ However, a nanowell is at the most 

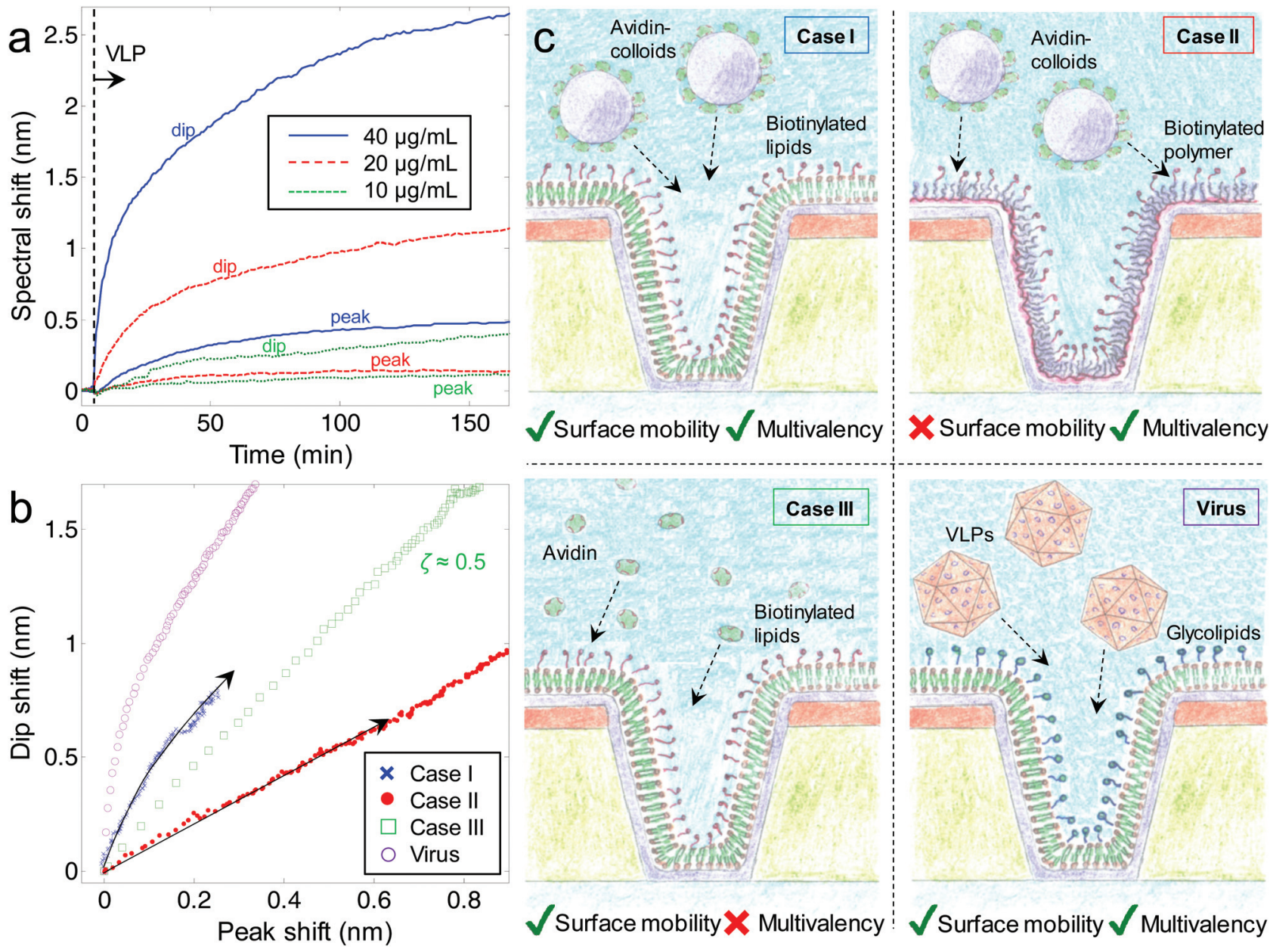

Fig. 3 VLP binding to nanowells and the influence from surface diffusivity and multivalency. Kinetics (dip and peak) of different concentrations of virus capsids binding to a membrane with glycosphingolipids following the contour of silica coated nanowells are shown in (a). The VLPs are introduced by an injection and binding monitored with stagnant solution. Scatter plots of peak and dip shifts for binding of avidin-coated particles, avidin and VLPs $\left(40 \mu \mathrm{g} \mathrm{mL}^{-1}\right)$ are shown in (b). Illustrations of the measured interactions using silica coated nanowells are shown in (c).

$100 \mathrm{~nm}$ away and the binding is over the timescale of hours (Fig. 3a). Indeed, using the location-specific detection, we found high $\zeta$ values $(\sim 0.9$ for low concentration and exposure for a few min, $0.8 \pm 0.05$ after $\sim 1 \mathrm{~h}$ ) for the VLP binding compared to other binding events (e.g. Fig. 2a). Further, $\zeta$ was higher for lower VLP concentrations and after shorter incubation time, showing that as the nanowells become occupied VLPs are forced to the planar surface instead. (Most of the projected surface area is planar.) Binding of VLPs inside nanowells was further supported by scanning electron microscopy imaging after binding (ESI $\dagger$ ).

To test if multivalency, surface diffusivity and size-matching were the reasons for VLP accumulation inside membrane coated nanowells, we used a model system with $\sim 50 \mathrm{~nm}$ silica particles covered with avidin (Fig. 3b). These particles can bind to multiple biotinylated lipids in a membrane (case I, Fig. 3c). As control, we let the same particles bind to biotinylated poly(ethylene glycols) grafted to a poly(L-lysine) backbone adsorbed on the silica covered nanowells, ${ }^{34}$ forming a layer similar in thickness to the lipid membrane (case II, Fig. 3c). This system provides multivalency but without the surface mobility of a fluid membrane. As another control, we let avidin bind to a membrane with biotinylated lipids (case III, Fig. 3c). This system provides surface mobility but no multivalency since the avidin tetramer has a maximum of two accessible binding sites. The results are summarized in Fig. $3 \mathrm{~b}$ as scatter plots of $\Delta \lambda_{\text {dip }} v s . \Delta \lambda_{\text {peak }}$ during binding. When the silica particles bind to biotinylated lipids in the membrane (case I), the dip shifts strongly while the peak shifts relatively little, especially in the initial part on the binding process ( $\zeta$ is time dependent). This proves spontaneous accumulation in nanowells, as further supported by scanning electron microscopy after binding (ESI $\dagger$ ). In contrast, when the same silica particles bind to a biotinylated ethylene glycol surface (case II) this generates a low $\zeta=0.16 \pm 0.05$, consistent with binding mainly on the planar surface. (Determined after $\sim 1 \mathrm{~h}$ of binding.) This is expected since without surface mobility, the colloids become stuck where they land by the irreversible biotin-avidin 
interaction. Since most of the exposed area is planar, the colloids are then unlikely to attach in the interior of the nanowells. As the much smaller avidin protein binds to biotinylated lipids (case III) there is a straight line with $\zeta=0.5 \pm 0.1$ as expected since the $(\sim 5 \mathrm{~nm})$ protein has no reason to achieve contact with more biotin receptors on the curved membrane. This makes avidin equally likely to be located anywhere on the membrane. The VLP binding generates the highest $\zeta$ and a clear curvature in the scatter plot which we attribute to the fact that the nanowells eventually become filled with VLP. Qualitatively the same behavior can be seen also for the model system with colloids, although their tendency to accumulate inside nanowells is lower, tentatively attributed to their slightly larger size. There may also be a small fraction of colloids that are not surface mobile and bound by nonspecific interactions.

\section{Conclusions}

We have shown how the concept of location-specific nanoplasmonic sensing by monitoring multiple spectral parameters can be used to answer questions related to how molecular binding is influenced by nanoscale geometry. The system presented here can be used to determine how the negative curvature in a lipid bilayer influences binding to membrane receptors, highlighting the importance of multivalency. ${ }^{35}$ The work represents a new type of application for nanoplasmonic sensors which is clearly not possible to achieve with conventional SPR or any other planar surface technique. Further, the methodology is very simple and the only instrumentation required is an ordinary spectrophotometer. Our results on VLPs indicate that beyond being able to induce membrane curvature, ${ }^{11}$ VLPs can also preferentially accumulate into existing nanometric invaginations on cells. In general, the information obtainable by our sensor should prove useful for understanding the norovirus infection mechanism and possibly the development of vaccines.

Future work with biomolecular interactions on membranes using this system can also further address the role of surface curvature on lipid domain formation and how this influences binding. ${ }^{31}$ In addition, one can also use our sensor for studies in the emerging research fields of how proteins ${ }^{12,13}$ and nanoparticles $^{35}$ respond to curved membranes (e.g. membrane budding, fusion and endocytosis). It is particularly straightforward to allow molecules of interest to access regions of negative curvature compared to work with giant vesicles. ${ }^{13}$ In fact, the concept of location-specific detection can also be applied to other systems beyond lipid membranes in order to study effects of negative surface curvature in general and localization of molecules in nanoscale containers.

\section{Notes and references}

1 A. B. Dahlin, N. J. Wittenberg, F. Hook and S.-H. Oh, Nanophotonics, 2013, 2, 83-101.
2 P. Zijlstra, P. M. R. Paulo and M. Orrit, Nat. Nanotechnol., 2012, 7, 379-382.

3 A. B. Dahlin, Sensors, 2012, 12, 3018-3036.

4 J. Homola, Chem. Rev., 2008, 108, 462-493.

5 R. L. Rich and D. G. Myszka, J. Mol. Recognit., 2011, 24, 892-914.

6 K. Kumar, A. B. Dahlin, T. Sannomiya, S. Kaufmann, L. Isa and E. Reimhult, Nano Lett., 2013, 13, 61226129.

7 E. C. Yusko, J. M. Johnson, S. Majd, P. Prangkio, R. C. Rollings, J. L. Li, J. Yang and M. Mayer, Nat. Nanotechnol., 2011, 6, 253-260.

8 N. S. Hatzakis, V. K. Bhatia, J. Larsen, K. L. Madsen, P. Y. Bolinger, A. H. Kunding, J. Castillo, U. Gether, P. Hedegard and D. Stamou, Nat. Chem. Biol., 2009, 5, 835841.

9 Y.-S. Ryu, I.-H. Lee, J.-H. Suh, S. C. Park, S. Oh, L. R. Jordan, N. J. Wittenberg, S.-H. Oh, N. L. Jeon, B. Lee, A. N. Parikh and S.-D. Lee, Nat. Commun., 2014, 5, 4507.

10 B. Sorre, A. Callan-Jones, J. Manzi, B. Goud, J. Prost, P. Bassereau and A. Roux, Proc. Natl. Acad. Sci. U. S. A., 2012, 109, 173-178.

11 G. E. Rydell, L. Svensson, G. Larson, L. Johannes and W. Romer, Biochim. Biophys. Acta, Biomembr., 2013, 1828, 1840-1845.

12 B. Antonny, Annu. Rev. Biochem., 2011, 80, 101-123.

13 P. Sens, L. Johannes and P. Bassereau, Curr. Opin. Cell Biol., 2008, 20, 476-482.

14 H. J. Wu, J. Henzie, W. C. Lin, C. Rhodes, Z. Li, E. Sartorel, J. Thorner, P. D. Yang and J. T. Groves, Nat. Methods, 2012, 9, 1189-1191.

15 J. Junesch, T. Sannomiya and A. B. Dahlin, ACS Nano, 2012, 6, 10405-10415.

16 P. Kukura, H. Ewers, C. Muller, A. Renn, A. Helenius and V. Sandoghdar, Nat. Methods, 2009, 6, 923-927.

17 J. A. Boga, S. Melon, I. Nicieza, I. de Diego, M. Villar, F. Parra and M. de Ona, J. Clin. Microbiol., 2004, 42, 26682674.

18 M. Bally, A. Gunnarsson, L. Svensson, G. Larson, V. P. Zhdanov and F. Hook, Phys. Rev. Lett., 2011, 107, 188103.

19 A. B. Dahlin, M. Mapar, K. L. Xiong, F. Mazzotta, F. Hook and T. Sannomiya, Adv. Opt. Mater., 2014, 2, 556564.

20 H. Im, N. J. Wittenberg, A. Lesuffleur, N. C. Lindquist and S. H. Oh, Chem. Sci., 2010, 1, 688-696.

21 A. B. Dahlin, Analyst, 2015, 140, 4748-4759.

22 T. Sannomiya, O. Scholder, K. Jefimovs, C. Hafner and A. B. Dahlin, Small, 2011, 7, 1653-1663.

23 A. B. Dahlin, J. O. Tegenfeldt and F. Hook, Anal. Chem., 2006, 78, 4416-4423.

24 R. Marie, A. B. Dahlin, J. O. Tegenfeldt and F. Hook, Biointerphases, 2007, 2, 49-55.

25 F. Mazzotta, T. W. Johnson, A. B. Dahlin, J. Shaver, S.-H. Oh and F. Hook, ACS Photonics, 2015, 2, 256-262. 
26 P. B. Johnson and R. W. Christy, Phys. Rev. B: Solid State, 1972, 6, 4370-4379.

27 M. P. Jonsson, P. Jonsson, A. B. Dahlin and F. Hook, Nano Lett., 2007, 7, 3462-3468.

28 G. H. Zan, J. A. Jackman, S. O. Kim and N. J. Cho, Small, 2014, 10, 4828-4832.

29 K. Kumar, L. Isa, A. Egner, R. Schmidt, M. Textor and E. Reimhult, Langmuir, 2011, 27, 10920-10928.

30 B. Fiege, C. Rademacher, J. Cartmell, P. I. Kitov, F. Parra and T. Peters, Angew. Chem., Int. Ed., 2012, 51, 928-932.
31 M. Bally, G. E. Rydell, R. Zahn, W. Nasir, C. Eggeling, M. E. Breimer, L. Svensson, F. Hook and G. Larson, Angew. Chem., Int. Ed., 2012, 51, 12020-12024.

32 J. Angstrom, K. E. Falk, K. A. Karlsson and G. Larson, Biochim. Biophys. Acta, 1982, 712, 274-282.

33 G. E. Rydell, A. B. Dahlin, F. Hook and G. Larson, Glycobiology, 2009, 19, 1176-1184.

34 N. P. Huang, J. Voros, S. M. De Paul, M. Textor and N. D. Spencer, Langmuir, 2002, 18, 220-230.

35 H.-M. Ding and Y.-Q. Ma, Small, 2015, 11, 1055-1071. 